551. 511.3

\title{
On the Truncation Errors in Numerical Prediction
}

\author{
by \\ M. Magata \\ Meteorological Research Institute
}

(Received July 12, 1957)

\begin{abstract}
The behaviour of the truncation errors in the numerical prediction using grid points, is discussed taking into account the scale of the perturbations.

When we forecast by the ordinary numerical prediction method the path of the relatively small cyclone such as the typhoon, the velocity of the center is known to become smaller than the observed value. We conclude that this fact can be explained by the behaviour of the truncation errors.
\end{abstract}

\section{Introduction}

In numerical weather prediction the vorticity equation is used in a finite difference form, and the behaviour of the truncation errors gives rise to discussion. So far as the largescale perturbations are concerned, the truncation errors are unimportant, but when we deal with relatively small-scale perturbations, they can't be ignored. For instance, in forecasting the path of the relatively smallscale cyclone such as the typhoon by the ordinary numerical prediction, the velocity of the center of the cyclone is known to be smaller than the observed value (IToo and MASUdA, 1957). In order to explain this point, when the motion is regarded as consisting of circular perturbation on a uniform current, we compared the solutions of the vorticity equation obtained by use of the finite difference method with analytically exact solutions. Further, by applying the method with which FLOHN and collaborators (1953) discussed the behaviour of truncation errors, it may be concluded that the above fact can be explained by the behaviour of truncation errors.

\section{General consideration}

We assume that the isobaric height distribution $Z$ may be expressed as follows:

$$
Z=a_{0}+\sum_{n=1}^{\infty}\left(a_{n} \cos \frac{n \pi}{a} x+b_{n} \sin \frac{n \pi}{a} y\right),
$$

where $a_{n}$ and $b_{n}$ are functions of $y$, and $x$ and $y$ are cartesian coordinates. Then the $y$-component $v$ of the wind velocity becomes

$$
v=\frac{g}{f} \frac{\partial Z}{\partial x}=\frac{g}{f} \sum_{n=1}^{\infty} \frac{n \pi}{a}\left(-a_{n} \sin \frac{n \pi}{a} x+b_{n} \cos \frac{n \pi}{a} y\right) .
$$


If we use $\frac{Z(x+d)-Z(x-d)}{d}$ in place of $\frac{\partial Z}{\partial x}$, then (2) becomes

$$
[v]=\frac{g}{f}\left[\frac{\partial Z}{\partial x}\right]=\frac{g}{f} \sum_{n=1}^{\infty} \frac{\sin \frac{n \pi}{a} d}{d}\left(-a_{n} \sin \frac{n \pi}{a} x+b_{n} \cos \frac{n \pi}{a} x\right),
$$

where $\left[\frac{\partial Z}{\partial x}\right]=\frac{Z(x+d)-Z(x-d)}{d}$, and $d$ is a gridsize.

Since $-\frac{n \pi}{a}<\frac{\sin \frac{n \pi}{a} d}{d}<\frac{n \pi}{a}$,
amplitude of each term of
$\left.\frac{\partial}{\partial x}\right]$ insteas of $\frac{\partial Z}{\partial x}$, only when

$$
\frac{\sin \frac{n \pi}{a} d}{d} \fallingdotseq \frac{n \pi}{a}
$$

On the other hand, if $n$ is sufficiently large for a fixed magnitude of $d$, we can not regard (4) as correct. Therefore when we use (3), we must take adequate $N$ such that

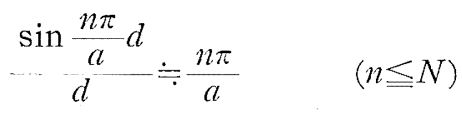

and consider the first $N$ terms of (2) and (3). In order to discuss the condition (4), we consider $Z$ to be expressed as follows :

$$
Z=A \sin \frac{2 \pi}{L} x,
$$

where $L$ is a wave-length. In this case

$$
\begin{gathered}
\frac{\partial Z}{\partial x}=\frac{2 \pi}{L} A \cos \frac{2 \pi}{L} x, \\
{\left[\frac{\partial Z}{\partial x}\right]=\frac{\sin \frac{2 \pi}{L} d}{d} A \cos \frac{2 \pi}{L} x .}
\end{gathered}
$$

We put

$$
\text { (6) } \sigma=\frac{\left[\frac{\partial Z}{\partial x}\right]}{\frac{\partial Z}{\partial x}}=\frac{L}{2 \pi d} \sin \frac{2 \pi}{L} d
$$

If we may regard $\sigma \fallingdotseq 1$, we can use $\left[\frac{\partial Z}{\partial x}\right]$ in place of $\frac{\partial Z}{\partial x}$. Fig. 1 shows the relation between $\sigma$ and the wavelength $L$. As may be seen from this figure, we can use $\left[\frac{\partial Z}{\partial x}\right]$ instead of $\frac{\partial Z}{\partial x}$

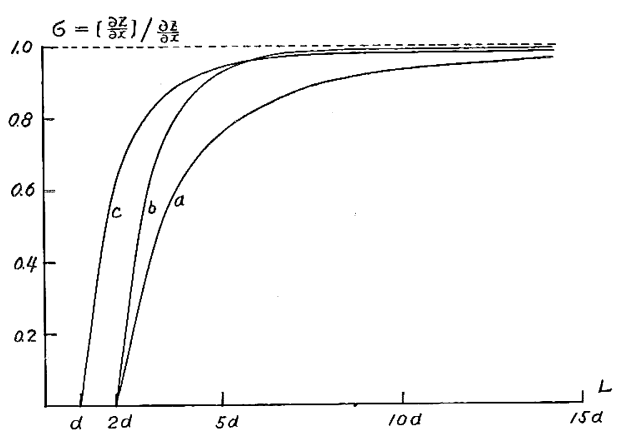

Fig. 1. The relation between the wave length $L$ and $\sigma=\left[\frac{\partial Z}{\partial x}\right] / \frac{\partial Z}{\partial x}$ for $Z=A \sin \frac{2 \pi}{L} x$.

(a) the case of $\left[\frac{\partial Z}{\partial x}\right]=\frac{Z(x+d)-Z(x-d)}{2 d}$ and $d=300 \mathrm{~km}$. (b) the case of $\left[\frac{\partial Z}{\partial x}\right]$ $=\frac{8(Z(x+d)-Z(x-d))-(Z(x+2 d)-Z(x-2 d))}{12 d}$ and $d=300 \mathrm{~km}$. (c) the case of $\left[\frac{\partial Z}{\partial x}\right]$ $=\frac{Z(x+d)-Z(x-d)}{2 d}$ and $d=150 \mathrm{~km}$. 
by the error less than $20 \%$ when the wave-length $L>6 d$. From the same consideration as above, we shall discuss how

$$
\left[\nabla^{2} Z\right]=\frac{Z(x+d, y)+Z(x-d, y)+Z(x, y+d)+Z(x, y-d)-4 Z(x, y)}{d^{2}}
$$

may be used in place of

$$
\nabla^{2} Z=\frac{\partial^{2} Z}{\partial x^{2}}+\frac{\partial^{2} Z}{\partial y^{2}}
$$

Let

$$
Z=A \sin \frac{2 \pi}{L} x \sin \frac{2 \pi}{D} y
$$

then

$$
\begin{gathered}
\Gamma^{2} Z=-\left(\frac{4 \pi^{2}}{L^{2}}+\frac{4 \pi^{2}}{D^{2}}\right) A \sin \frac{2 \pi}{L} x \sin \frac{2 \pi}{D} y . \\
{\left[\nabla^{2} Z\right]=-\frac{2}{d^{2}}\left(2-\cos \frac{2 \pi}{L} d-\cos \frac{2 \pi}{D} d\right) A \sin \frac{2 \pi}{L} x \sin \frac{2 \pi}{D} y .}
\end{gathered}
$$

(8) and (9) differ only in amplitude and have the same form in $x$ and $y$.

Let

$$
\sigma_{m n}=\frac{\left[\nabla^{2} Z\right]}{\nabla^{2} Z}=\frac{2-\cos \frac{2 \pi}{m}-\cos \frac{2 \pi}{n}}{2 \pi^{2}\left(\frac{1}{m^{2}}+\frac{1}{n^{2}}\right)},
$$

where

$$
L=m d \text { and } D=n d \text {. }
$$

Then if we may regard $\sigma_{m n} \doteqdot 1$, we can use $\left[\nabla^{2} Z\right]$ in place of $\nabla^{2} Z$. Fig. 2 shows the relations between $\sigma_{m n}$ and the wave-numbers. As may be seen from this figure, when $L$ and $D$ are larger than $4 d$, the truncation errors are less than $20 \%$. And the amplitude of $\left[\nabla^{2} Z\right]$ is always smaller than that of $\nabla^{2} Z$. Conversely we shall consider the poisson equation as follows:

$$
\nabla^{2} \psi=A \sin \frac{2 \pi}{L} x \sin \frac{2 \pi}{D} y,
$$

Writing this equation in a difference form,

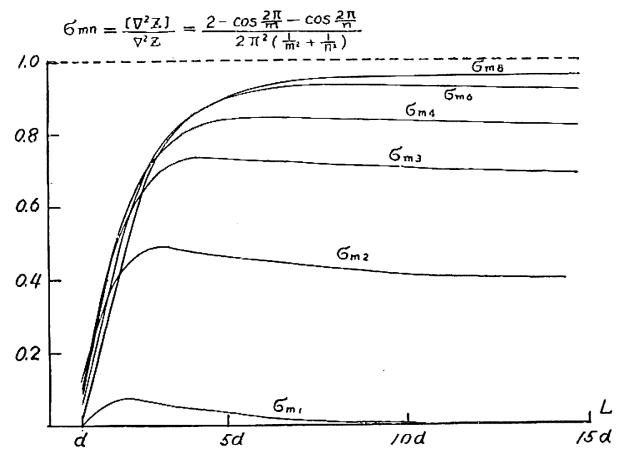

Fig. 2. The relation between the wavenumbers and

$$
\sigma_{m n}=\frac{\left[\Gamma^{2} Z\right]}{\Gamma^{2} Z}=\frac{2-\cos 2 \pi / m-\cos 2 \pi / n}{2 \pi^{2}\left(1 / m^{2}+1 / n^{2}\right)},
$$
for $Z=A \sin \frac{2 \pi}{L} x \sin \frac{2 \pi}{D} y$ where $L=m d$ and $D=n d$.

$$
\left[\nabla^{2} \phi\right]=A \sin \frac{2 \pi}{L} x \sin \frac{2 \pi}{D} y .
$$

In this case, the solution of (12) is larger in amplitude than that of (11). Next, the barotropic vorticity equation may be written 
(13)

$$
\frac{\partial}{\partial t} \nabla^{2} Z=J\left(\frac{g}{f} \nabla^{2} Z+f, Z\right)
$$

where

$$
J(A, B)=\frac{\partial A}{\partial x} \quad \frac{\partial B}{\partial y}-\frac{\partial A}{\partial y} \frac{\partial B}{\partial x} .
$$

We shall estimate the truncation errors in calculating $J\left(\nabla^{2} Z, Z\right)$.

Let the height $Z$ be given as follows:

$$
Z=\sum_{m, n=1}^{\infty} A_{m n} \sin \frac{m \pi}{a} x \sin \frac{n \pi}{a} y,
$$

then

$$
J\left(\nabla^{2} Z, Z\right)=\sum_{\substack{m, n=1 \\ k, l=1}}^{\infty} J\left(A_{m n} \sin \frac{m \pi}{a} x \sin \frac{n \pi}{a} y, A_{k l} \sin \frac{k \pi}{a} x \sin \frac{l \pi}{a} y\right) .
$$

And therefore we shall discuss the truncation errors in computation of $J\left(\nabla^{2} Z_{1}, Z_{2}\right)$, where

$$
\begin{aligned}
& Z_{1}=A \sin \frac{2 \pi}{K} x \sin \frac{2 \pi}{L} y, \\
& Z_{2}=B \sin \frac{2 \pi}{M} x \sin \frac{2 \pi}{N} y,
\end{aligned}
$$

Calculating $J\left(\nabla^{2} Z_{1}, Z_{2}\right)$

$$
\begin{aligned}
J\left(\nabla^{2} Z_{1}, Z_{2}\right)= & 4 \pi^{2}\left(\frac{4 \pi^{2}}{K^{2}}+\frac{4 \pi^{2}}{L^{2}}\right) A B \\
& \times\left\{\frac{1}{L M} \sin \frac{2 \pi}{K} x \cos \frac{2 \pi}{M} x \sin \frac{2 \pi}{N} y \cos \frac{2 \pi}{L} y\right. \\
& \left.-\frac{1}{K N} \sin \frac{2 \pi}{M} x \cos \frac{2 \pi}{K} x \sin \frac{2 \pi}{L} y \cos \frac{2 \pi}{N^{-}} y\right\} .
\end{aligned}
$$

If we assume $\left[J\left(\nabla^{2} Z_{1}, Z_{2}\right)\right]$ to be value of $J\left(\nabla^{2} Z_{1}, Z_{2}\right)$ calculated using gridsize $d$, then

$$
\begin{aligned}
{\left[J\left(\nabla^{2} Z_{1}, Z_{2}\right)\right]=} & \frac{2}{d^{2}}\left(2-\cos \frac{2 \pi}{K} d-\cos \frac{2 \pi}{L} d\right) A B \\
& \times\left\{\frac{\sin \frac{2 \pi}{L} d \sin \frac{2 \pi}{M} d}{d^{2}} \sin \frac{2 \pi}{K} x \cos \frac{2 \pi}{M} x \sin \frac{2 \pi}{N} y \cos \frac{2 \pi}{L} y\right. \\
& \left.-\frac{\sin \frac{2 \pi}{K} d \sin \frac{2 \pi}{N} d}{d^{2}} \sin \frac{2 \pi}{M} x \cos \frac{2 \pi}{K} x \sin \frac{2 \pi}{L} y \cos \frac{2 \pi}{N} y\right\}
\end{aligned}
$$

Let $\gamma$ be a ratio of the first term of (15) to that of (14), then

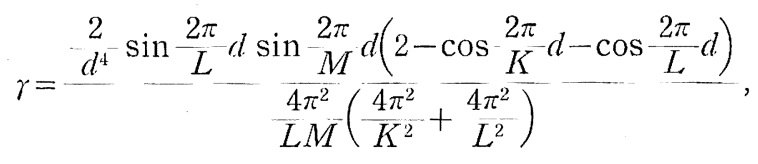


Fig. 3 shows the relation between $\gamma$ and the wave-length when $K=L$ and $d=300 \mathrm{~km}$. In this case the truncation errors are larger than in the case of Fig. 1 and less than 20\% when the wave-length $L>8 d$.

\section{Numerical prediction of the path of the typhoon}

According to the study of IToo and MASUdA (1957), when the path of the typhoon is forecasted by the ordinary numerical prediction method, the velocity of the center is smaller than the observed

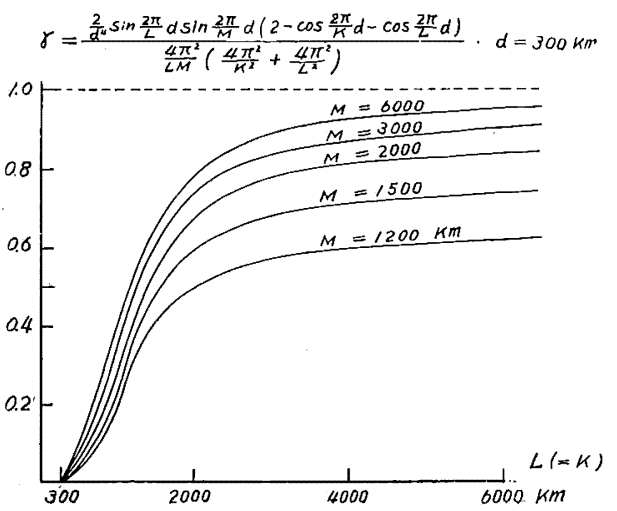

Fig. 3. The relation between

$$
\gamma=\frac{2 / d^{4} \sin \frac{2 \pi}{L} d \sin \frac{2 \pi}{M} d\left(2-\cos \frac{2 \pi}{K} d-\cos \frac{2 \pi}{L} d\right)}{4 \pi^{2} / L M\left(4 \pi^{2} / K^{2}+4 \pi^{2} / L^{2}\right)}
$$

and the wave length when $K=L$ and $d=300 \mathrm{~km}$. value. In order to clarify this point, we shall discuss in this section the movement of the circular disturbance on a uniform current and the truncation errors in this case. Assuming that the motion is regarded as consisting of circular disturbance on a uniform current, an initial height distribution $Z_{0}$ may be written as follows:

$$
Z_{0}=C-\frac{f}{g} U y+F(r),
$$

where $C=$ const, $U$ uniform velocity and $F(r)$ is a function of $r=\sqrt{x^{2}+y^{2}}$. Then the solution of the barotropic vorticity equation (13) becomes

$$
Z=C-\frac{f}{g} U y+F\left(\sqrt{(x-c t)^{2}+y^{2}}\right) .
$$

On the other hand, above $Z$ there is the following relation:

$$
J\left(\frac{g}{f} \nabla^{2} Z+f, Z\right)=-U \frac{\partial}{\partial x} \nabla^{2} F .
$$

And therefore (13) may be written

$$
\frac{\partial}{\partial t} \nabla^{2} F=-U \frac{\partial}{\partial x} \nabla^{2} F
$$

so that

$$
\frac{\partial F}{\partial t}=-U \frac{\partial F}{\partial x} .
$$

The solution of (19) becomes

$$
F=F\left(\sqrt{(x-U t)^{2}+y^{2}}\right)
$$

Namely, the initial pattern moves without changing the shape with the same 
velocity as the uniform flow. Then we shall replace (19) with a difference equation using a gridsize $d$. We assume $F(r)$ to be given by the following:

$$
F(r)=-\frac{\delta}{2}\left(e^{-\frac{r^{2}}{l^{2}}}+e^{-\frac{r^{2}}{L^{2}}}\right) \text {. }
$$

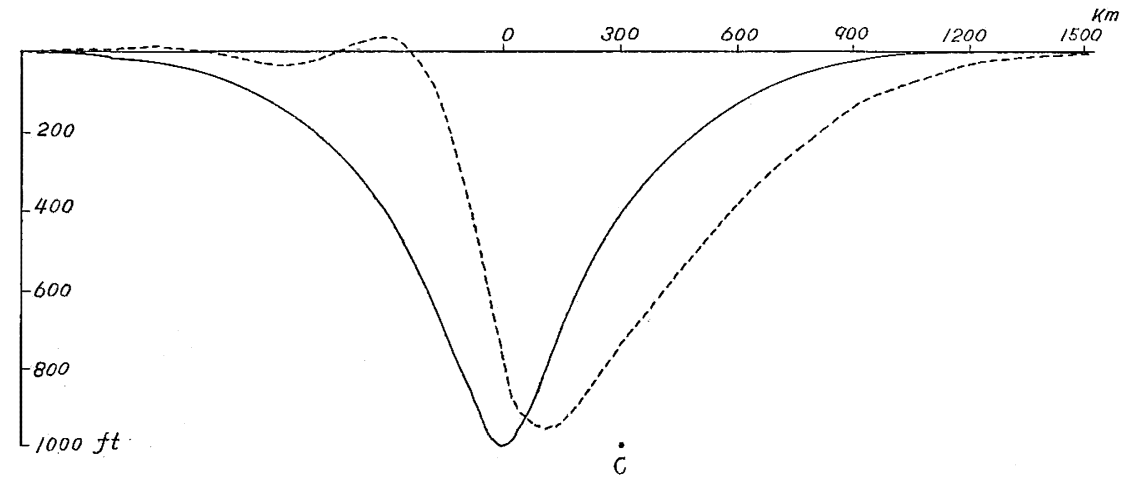

Fig. 4. Full line is the distribution of $F(r)=-\frac{\partial}{2}\left(e^{-r^{2}} l^{2}+e^{-\frac{r^{2}}{L^{2}}}\right)$ when $\delta=1,000 \mathrm{ft}, l=200 \mathrm{~km}, L=500 \mathrm{~km}$ and $t=0$. Dashed line is the distribution of $F(r)$ in $t=12 \mathrm{hr}$ calculated by 12 time steps of $\Delta t=1 \mathrm{hr}$. Point $C$ shows the location of the center of the exact solution in $t=12 \mathrm{hr}$.

In Fig. 4, the full line shows the distribution (21) when $\delta=1,000 \mathrm{ft}, l=200 \mathrm{~km}$ and $L=500 \mathrm{~km}$. We shall calculate on $y=0$ only and use $F(x)$ in place of $F(r)$. In order to solve (19) using grid points, we calculate $\frac{F(x+d)-F(x-d)}{2 d}$ instead of $\frac{\partial F}{\partial x}$ where $d$ is a gridsize of $300 \mathrm{~km}$ and put

$$
(F)_{t=\Delta t}=(F)_{t=0}+\left(\frac{\partial F}{\partial t}\right)_{t=0} \Delta t=\left(F^{\prime}\right)_{t=0}-U \frac{F(x+d)-F(x-d)}{2 d} \Delta t .
$$

Thus the distribution of $F$ in $t=\Delta t$ can be obtained. Next the distribution of $F$ in $t=2 \Delta t, 3 \Delta t$, can be obtained as follows :

$$
\begin{aligned}
& (F)_{t=2 \Delta t}=(F)_{t=0}+2 \Delta t\left(\frac{\partial F}{\partial t}\right)_{t=\Delta t} \\
& (F)_{t=3 \Delta t}=(F)_{t=\Delta t}+2 \Delta t\left(\frac{\partial F}{\partial t}\right)_{t=2 \Delta t}
\end{aligned}
$$

In Fig. 4, the dashed line shows the distributions of $F$ in $t=12 \mathrm{hr}$ when in the above method we put $\Delta t=1$, and point $C$ is the location of the center of the exact solution of (19) when $t=12 \mathrm{hr}$. As may be seen from this figure, the velocity of the center when we used grid points is smaller than the actual velocity.

Next, if we expand (21) into a double fourier series in $-a \leqq x \leqq a$ and put $y=0$, we have (assuming wave number $\leqq 3$ )

$$
F(x)=-101-187 \cos \frac{\pi}{a} x-141 \cos \frac{2 \pi}{a} x-94 \cos \frac{3 \pi}{a} x
$$




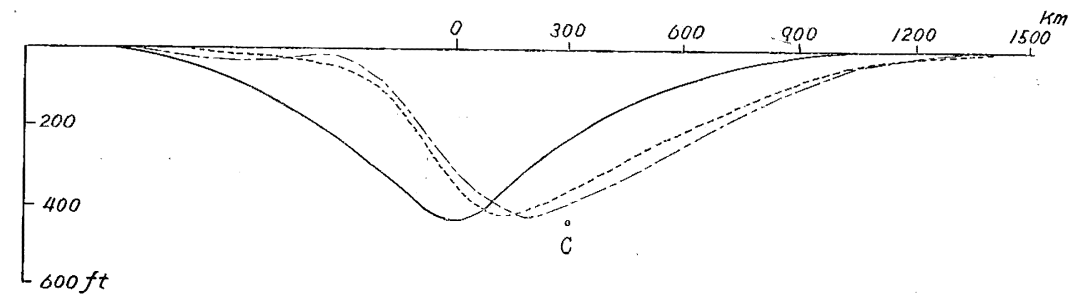

Fig. 5. Full line is the distribution of the stream function $\phi(r)$ in $t=0$. Dashed line is the distribution of $\phi(r)$ in $t=12 \mathrm{hr}$ calculated by 12 time steps of $\Delta t=1 \mathrm{hr}$. Chain line is the distribution of $\phi(r)$ in $t=12 \mathrm{hr}$ calculated by 12 time steps of $\Delta t=1 \mathrm{hr}$ when (34) was used. Point $C$ shows the location of the center of the exact solution in $t=12 \mathrm{hr}$.

where $a=2,400 \mathrm{~km}$. In Fig. 6, the full line shows the distribution of $F(x)$ given by (22) and the dashed line shows the distribution of $F$ in $t=12 \mathrm{hr}$, calculated using grid points as above. In this case the velocity of the center of $F$ is the nearly exact value. It may be considered that in this case the truncation errors are removed because the relatively small scale wave is cut out.

Next we shall use a stream function $\phi$ instead of the height $Z$. In the case of the axial symmetric pattern the relation between the stream function $\phi$ and the height $Z$ can be given by the so-called gradient wind equation

$$
\left(\frac{d \psi}{d r}\right)^{2}+f r-d \psi=g r \frac{d Z}{d r}
$$

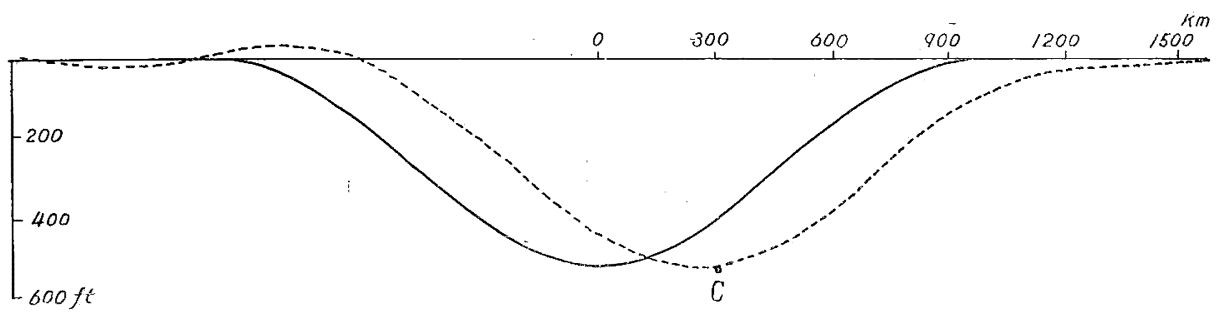

Fig. 6. Full line is the distribution of

$$
\begin{aligned}
& F(x)=-101-187 \cos \frac{\pi}{a} x-141 \cos \frac{2 \pi}{a} x-94 \cos \frac{3 \pi}{a} x \text { in } t=0 . \text { Dashed } \\
& \text { line is the distribution of } F(x) \text { in } t=12 \mathrm{hr} \text { calculated by } 12 \text { time } \\
& \text { steps of } \Delta t=1 \mathrm{hr} \text {. Point } C \text { shows the location of the center of the } \\
& \text { exact solution in } t=12 \mathrm{hr} \text {. }
\end{aligned}
$$

In Fig. 5, the full line shows the distribution of the stream function $\phi$ for the height $Z$ given by (21) and the dashed line shows the distribution of $\phi$ in $t=12 \mathrm{hr}$ calculated using grid point, for the initial pattern. In this case the velocity of the center is about $50 \%$ of the actual value. Thus it may be considered that in numerical prediction the calculated velocity of the typhoon becomes smaller than the observed value due to the truncation errors. In order to clarify this point, in the next section we shall discuss the truncation errors of the solution of (19) in the case of the sinusoidal perturbation of various wave-length. 
4. The behaviour of truncation errors when the solutions are sine waves If we put

$$
\begin{aligned}
t & =m \Delta t, \quad x \\
& =n \Delta x \\
F(t, x) & =F(m \Delta t, n \Delta x)=F_{m n} \quad(m, n=0, \pm 1, \pm 2, \cdots \cdots)
\end{aligned}
$$

then (19) becomes, writing in finite difference form,

$$
\frac{F_{m+1 n}-F_{m-1 n}}{2 \Delta t}=-U \frac{F_{m n+1}-F_{m n-1}}{2 \Delta x},
$$

where we use central finite differences. We assume

$$
F_{m n}=A e^{i^{2-\frac{\pi}{L}}(n \Delta x-C m \Delta t)}
$$

and if this is substituted into (24) and keep in good shape, the relation between the wave velocity $c$ and the wave length $L$ may be obtained as follows:

$$
\sin \left(\frac{2 \pi c}{L} \Delta t\right)=U \frac{\Delta t}{\Delta x} \sin \left(\frac{2 \pi}{L} \Delta x\right) .
$$

Now we put

$$
\alpha \equiv U \frac{\Delta t}{\Delta x} \sin \left(\frac{2 \pi}{L} \Delta x\right)
$$

Thus when $|\alpha| \leqq 1$, we obtain the real velocity $c$ as follows :

$$
C=\frac{L}{2 \pi \Delta t} \sin ^{-1} \alpha
$$

On the other hand when $|\alpha|>1$, c being a complex quantity $c=c_{r}+i c_{i}$ results from (26)

$$
C_{r}=\frac{4 n+1}{4} \frac{L}{\Delta t} \quad(n=0, \pm 1, \pm 2, \cdots \cdots)
$$

$$
C_{i}=\frac{L}{2 \pi \Delta t} \cosh ^{-1}|\alpha| \text {. }
$$

Thus when $|\alpha| \leqq 1$

$$
F=A e^{i \frac{2 \pi}{L}\left(n \Delta x-C_{m} \Delta t\right)}
$$

where $c$ is given by (28). On the other hand when $|\alpha|>1$

$$
\left.F=A e^{\frac{2 \pi C_{i}}{L} m \Delta t} e^{i \frac{2 \pi}{L}(n \Delta x}-C_{r m}^{m \Delta} t\right),
$$

where $c_{r}$ and $c_{i}$ are given by (29) and (30).

And then the exact sinusoidal solution of (19) becomes as follows:

$$
F=A e^{i-\frac{2 \pi}{L}(x-U t)} .
$$


When $|\alpha| \leqq 1$, as may be seen from (31) and (33), the solution of the difference eqation (24) is the same form as the precise solution of the differential equation (19), but there is a discrepancy between the wave velocity $c$ and $U$. On the other hand, when $|\alpha|>1$, the solution (37) of the difference equation (24) is an unstable wave. This expresses the so-called computational instability. Fig. 7 shows the relation between $\frac{c}{U}$ and $\Delta t$ for various wave-lengths when $|\alpha| \leqq 1$. As may be seen from this figure, for a fixed time interval $\Delta t$, the wave velocity is small as the wave length is short. For instance, when $\Delta t=1 \mathrm{hr}, \frac{c}{U}$ are about, $0.41,0.64,0.75,0.82$ and 0.86 for the wave-lengths $L=900 \mathrm{~km}, 1,200 \mathrm{~km}$, $1,500 \mathrm{~km}, 1,800 \mathrm{~km}$ and $2,100 \mathrm{~km}$ respectively. It may be considered that this is the reason why the velocity of the axial symmetric pattern is smaller than the actual value in Fig. 4. In Fig. 6, since small-scall perturbations are

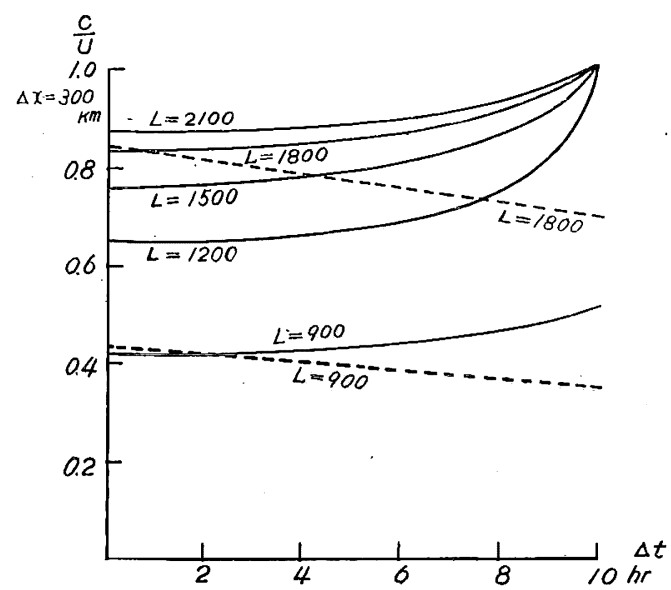

Fig. 7. The relation between $\frac{c}{U}$ and $\Delta t$ for various wave lengths when $|\alpha| \leqq 1$. Full lines are the case using central finite time differences. Dashed lines are the case using uncentered forward time differences. removed, the velocity of the center

is nearly equal to the precise one. It may be expected that the truncation errors become small by using a small gridsize or applying the accurate difference formula. Thus we shall use

$$
\frac{\partial Z}{\partial x} \doteqdot \frac{8(Z(x+d)-Z(x-d))-(Z(x+2 d)-Z(x-2 d))}{12 d},
$$

in place of

$$
\frac{\partial Z}{\partial x} \fallingdotseq \frac{Z(x+d)-Z(x-d)}{2 d}
$$

In Fig. 5, the chain line shows calculated value by 12 time steps of $\Delta t=1 \mathrm{hr}$ when we used (34). In this case, the center of the pattern moves more accurately than when (35) was used. On the other hand, using (34) the differential equation (19) can be rewritten as follows:

$$
\frac{F_{m+1 n}-F_{m-1 n}}{2 \Delta t}=-U-\frac{8\left(F_{m n+1}-F_{m n-1}\right)-\left(F_{m n+2}-F_{m n-2}\right)}{12 \Delta x} .
$$

If we substitute (25) into (36) and keep in good shape, the relation between the wave-velocity $c$ and the wave-length $L$ may be obtained as follows:

$$
\sin \left(\frac{2 \pi c}{L} \Delta t\right)=U \frac{\Delta t}{\Delta x} \frac{8 \sin \left(\frac{2 \pi}{L} \Delta x\right)-\sin \left(\frac{4 \pi}{L} \Delta x\right)}{6} .
$$


If $\left|U \frac{\Delta t}{\Delta x} \frac{8 \sin \left(\frac{2 \pi}{L} \Delta x\right)-\sin \left(\frac{4 \pi}{L} \Delta x\right)}{6}\right| \leqq 1$, then the wave-velocity $c$ becomes as follows

$$
C=\frac{L}{2 \pi: \Delta t} \sin ^{-1}\left(U \frac{\Delta t}{\Delta x} \frac{8 \sin \left(\frac{2 \pi}{L} \Delta x\right)-\sin \left(\frac{4 \pi}{L} \Delta x\right)}{6}\right) .
$$

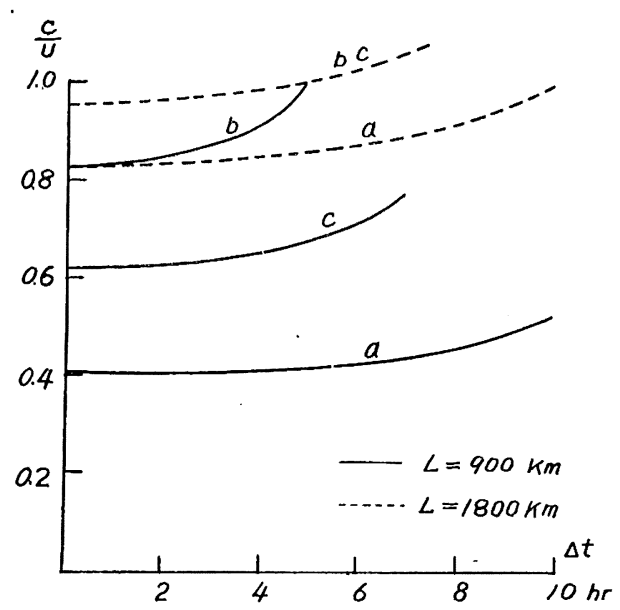

Fig. 8. The relation between $\frac{c}{U}$ and $\Delta t$.

(a) the case using the three points system (35) and $d=300 \mathrm{~km}$. (b) the case using the three points system (35) and $d=150 \mathrm{~km}$. (c) the case using the five points system (34) and $d=300 \mathrm{~km}$.
Fig. 8 shows the relation between $\frac{c}{U}$ and $\Delta t$ when (28) and (38) with $\Delta x=300 \mathrm{~km}$ were used for $L=900 \mathrm{~km}$ and $L=1,800 \mathrm{~km}$, and also the relation between $\frac{c}{U}$ and $\Delta t$ when (28) with $\Delta x=$ $150 \mathrm{~km}$ were used for $L=900 \mathrm{~km}$ and $L=1,800 \mathrm{~km}$. As may be seen from this figure (28) with $\Delta x=150 \mathrm{~km}$ and (38) with $\Delta x=300 \mathrm{~km}$ give a more precise wave-velocity.

Up to now we have used central finite time differences. Next we shall discuss the case using uncentered forward directed time differences. In this case the difference equation corresponding to (19) with uncentered differences becomes

$$
\frac{F_{m+1 n}-F_{m n}}{\Delta t}=-U \frac{F_{m n+1}-F_{m n-1}}{2 \Delta x}
$$

If we substitute (31) into (39), there is no real value of $c$ and the solution of (39) becomes as follows :

$$
F=\left(1+\alpha^{2}\right)^{\frac{m}{2}} A e^{i \frac{2 \pi}{L}\left(n \Delta x-c_{r} m \Delta t\right)}
$$

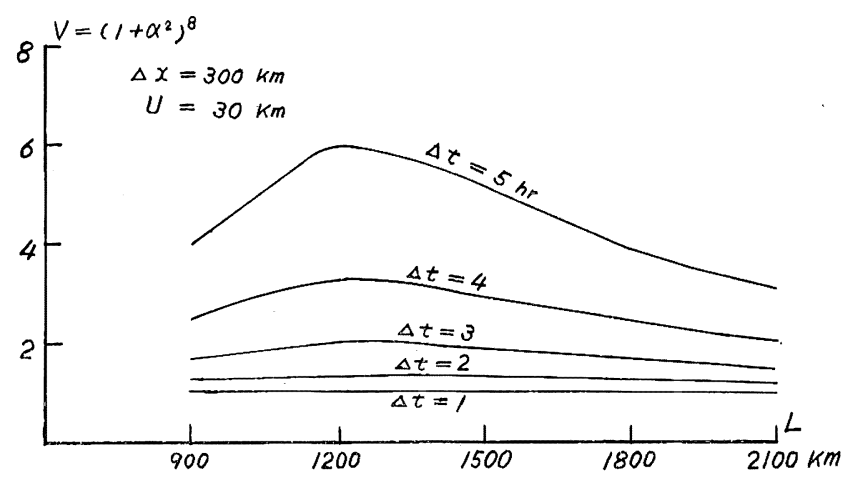

Fig. 9. The relation between the rate of the computational instability and wave length for various time intervals. 
where

$$
\begin{gathered}
C_{r}=\frac{L}{2 \pi \Delta t} \tan ^{-1}\left(U \frac{\Delta t}{\Delta x} \sin \left(\frac{2 \pi}{L} \Delta x\right)\right), \\
\alpha=U \cdot \frac{\Delta t}{\Delta x} \sin \left(\frac{2 \pi}{L} \Delta x\right) .
\end{gathered}
$$

(40) is an unstable wave. Fig. 9 shows the relation between the rate of instability and the wave-length for various time intervals. As may be seen from this figure, the above computational instability does not give rise to discussion so far as we discuss the $24 \mathrm{hr}$ numerical forecast using time intervals $\Delta t=1$ or $2 \mathrm{hr}$. In Fig. 7 the dashed line shows the relation between the wave-velocity of (40) and the wave-length $L$.

Acknowledgements-C. The writer wishes to express his hearty thanks to Dr. H. Arakawa and Dr. S. Syono for their guidance and to the staff members of N.W.P. group in Tokyo for their valuable suggestions and discussions.

\section{References}

Frohn, H. and Collaborators, 1954 : Research on objective weather forecasting. Final Report. Contract No. AF $61(514)-434$.

Iтоo, H. and $Y$. MASUdA, 1957: The numerical prediction of the typhoon movement by the relay computer, Pap. Met. Geophys., 7, 377-389. 